\title{
Intracranial Angioplasty with Enterprise Stent for Intracranial Atherosclerotic Stenosis: A Single-Center Experience and a Systematic Review
}

\author{
Bowen Sun $\mathbb{D}^{1},{ }^{1}$ Chao Xu $\mathbb{D},{ }^{1}$ Pei Wu $\mathbb{D},{ }^{1}$ Man Li $\mathbb{D},{ }^{2}$ Shancai Xu $\mathbb{D},{ }^{1}$ Chunlei Wang $\mathbb{D},{ }^{1}$

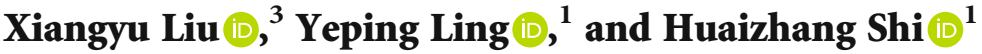 \\ ${ }^{1}$ Department of Neurosurgery, The First Affiliated Hospital of Harbin Medical University, Harbin, Heilongjiang Province, China \\ ${ }^{2}$ Department of Neurology, The First Affiliated Hospital of Harbin Medical University, Harbin, Heilongjiang Province, China \\ ${ }^{3}$ Department of Neurology, Shenzhen Longhua District Central Hospital, Shenzhen, Guangdong Province, China \\ Correspondence should be addressed to Huaizhang Shi; shihuaizhang@hrbmu.edu.cn
}

Received 3 November 2020; Revised 16 March 2021; Accepted 26 March 2021; Published 19 April 2021

Academic Editor: Xing Chen

Copyright (C) 2021 Bowen Sun et al. This is an open access article distributed under the Creative Commons Attribution License, which permits unrestricted use, distribution, and reproduction in any medium, provided the original work is properly cited.

\begin{abstract}
Background. The high rate of periprocedural complications for the endovascular stent procedure in the Stenting Versus Aggressive Medical Management Therapy for Intracranial Arterial Stenosis (SAMMPRIS) trial resulted in it being less recommended than medical therapy to treat intracranial atherosclerotic stenosis (ICAS). Because Enterprise stent use might reduce the incidence of complications in ICAS treatment compared to other frequently used stents, this paper evaluated the safety and effectiveness of the Enterprise stent for the treatment of ICAS. Methods. We performed a comprehensive literature search for reports on intracranial angioplasty using the Enterprise stent for ICAS treatment from the earliest date available from each database to May 2020 for PubMed, EMBASE, Web of Science, Cochrane, and Clinical Trials databases. We also reviewed the single-center experience of the First Affiliated Hospital of Harbin Medical University. We extracted information regarding periprocedural complications, procedure-related morbidity, mortality, immediate angiographic outcome, and long-term clinical and angiographic outcomes, among others. Event rates were pooled across studies using random-effects or fixed-effects models depending on the heterogeneity. Results. Five hundred fifty-seven patients with 588 lesions from seven studies, including the institutional series, were included in the analysis. The incidence of stroke or death within 30 days was $7.4 \%$ (95\% confidence interval (CI), 5.5\%-10.1\%). The incidence of ischemic stroke or TIA in the territory of the qualifying artery beyond 30 days and during follow-up was 3.2\% (95\% CI, 1.1\%-9.5\%). The incidence of in-stent restenosis was $10.1 \%$ (95\% CI, 4.6\%-22.2\%), and the incidence of symptomatic restenosis was $4.1 \%$ (95\% CI, 1.7\%-9.9\%). Conclusions. Intracranial angioplasty utilizing the Enterprise stent for ICAS treatment was relatively safe and effective but required further verification using additional sources for evidence.
\end{abstract}

\section{Introduction}

For decades, intracranial atherosclerotic stenosis (ICAS) has been a major risk factor for ischemic stroke worldwide, especially in Asian populations $[1,2]$. The Chinese Intracranial Atherosclerosis (CICAS) trial reported that, in China, $46.6 \%$ of patients with cerebral ischemia symptoms exhibited ICAS [3]. According to current guidelines, the primary treatment for ICAS is medical therapy [4]. However, for ICAS patients with severe stenosis and symptoms of cerebral ische- mia $(>70 \%)$, the stroke recurrence rate after receiving aggressive medical therapy (AMT) exceeds 20\% annually [5]. Percutaneous transluminal angioplasty and stenting (PTAS) has been regarded as an effective alternative method to treat severe ICAS [6].

In the past decade, two large randomized controlled trials (RCTs), Stenting Versus Aggressive Medical Management Therapy for Intracranial Arterial Stenosis (SAMMPRIS) [7] and Vitesse Intracranial Stent Study for Ischemic Stroke Therapy (VISSIT) [8], indicated that AMT was the preferred 
ICAS treatment relative to PTAS due to high rates of periprocedural complications associated with PTAS. In the SAMMPRIS trial, the 30-day incidence of stroke or death in the stent group was $14.7 \%$, which was significantly higher than $5.8 \%$ for the AMT group and was partly due to the use of the Wingspan stent $[9,10]$. The Wingspan stent is the only stent currently approved by the Food and Drug Administration for the treatment of ICAS [11]. However, concerns have been raised that its rigidity and open-cell design with high radial force could be related to the higher perioperative complication rate observed in previous trials [12].

Meanwhile, other stent varieties not originally designed to treat ICAS have been used for off-label treatment of ICAS. Some of these off-label stents have achieved satisfactory results, including other self-expanding stents such as the Enterprise stent (Codman Neurovascular, Raynham, Massachusetts, USA), balloon-expandable stents, and drug-eluting stents [9, 13-20]. Among these varieties, the Enterprise stent, which has a closed-cell design, special bearing system, and lower radial force, provides an attractive option to treat ICAS $[9,13-17]$. This preference is supported by the fact that the Enterprise stent is associated with fewer perioperative complications, and it can reach many lesions that other stents cannot due to the inclusion of microcatheters [9, 13-17]. However, only a few case series with limited sample sizes have been published that assess the treatment of ICAS using the Enterprise stent [9, 13-17]. No systematic review has been conducted. Due to the advantages mentioned above, it is possible that the Enterprise stent might become one of the predominant devices used to treat ICAS. Therefore, it is necessary to further verify the safety and effectiveness of the Enterprise stent in the treatment of ICAS. Thus, we reported on the outcomes of using the Enterprise stent to treat ICAS in a high-volume center and systematically reviewed all relevant literature.

\section{Materials and Methods}

\subsection{Institutional Series}

2.1.1. Patient Population and Lesion Characteristics. We conducted a study of case series from a single center and reported the results according to the Preferred Reporting Of CasE Series in Surgery (PROCESS) guidelines [21]. We retrospectively collected data from consecutive patients who underwent PTAS using the Enterprise Stent to treat ICAS in our institution (First Affiliated Hospital of Harbin Medical University, Harbin, China) from June 13, 2017, to April 3, 2020. The First Affiliated Hospital of Harbin Medical University is located in Harbin, Heilongjiang Province, in northeastern China. The area is located at a high altitude, cold climate, and a high incidence of cerebrovascular diseases. The center sees more than 1,500 cases of cerebrovascular diseases each year, including PTAS, and treats approximately 200 ICAS patients per year.

Written informed consent was obtained from each patient, and all study procedures were approved by the institutional ethics review board (Ethical approval: number ID, 20D0051). The inclusion criteria included the following. (1)
The patient received a diagnosis of ICAS with a degree $>70$ $\%$, which was confirmed by digital subtraction angiography (DSA), using the same methods as the Comparison of Warfarin and Aspirin for Symptomatic Intracranial Arterial Stenosis (WASID) trial [5]. (2) The patient exhibited recurrent transient ischemic attack (TIA) or ischemic stroke despite receiving AMT. (3) Hypoperfusion was present in the region surrounding the qualifying artery that was confirmed by computed tomography perfusion (CTP) and magnetic resonance imaging (MRI). The exclusion criteria included the presence of (1) complete occlusion of the cerebral artery; (2) stenosis caused by nonatherosclerotic factors; (3) ICAS combined with other intracranial diseases such as cerebral hemorrhage, malformation, aneurysm, moyamoya disease, and intracranial tumors; and (4) occurrence of a new ischemic stroke within two weeks before admission, as verified by diffusion-weighted imaging (DWI).

2.1.2. Procedures. All patients received dual antithrombotic therapy for a minimum of five days before stenting, as well as oral aspirin $(100 \mathrm{mg} / \mathrm{d})$ and clopidogrel $(75 \mathrm{mg} / \mathrm{d})$, and their platelet inhibition rate was assessed. Preoperative blood pressure was maintained 15\% lower than the baseline blood pressure. Patients remained under general anesthesia during the stent procedure, and heparin was infused following induction of anesthesia. A $6 \mathrm{~F}$ catheter was inserted into the common carotid or vertebral artery via the femoral artery using the Seldinger method. After femoral artery puncture, heparin was injected intravenously, and heparin saline was continuously infused during the procedure to ensure that the activated clotting time was between 150 and 250 seconds. Subsequently, angiography was performed to assess stenosis lesions and blood flow compensation.

We calculated the diameter and length of stenosis lesions utilizing both three-dimensional rotation and twodimensional imaging. Under the guidance of the path map, a Traxcess 14 (Microvention, USA) microguidewire was used with the Excelsior SL-10 (Stryker, Kalamazoo, Michigan, USA) microcatheter to super select the distal end or branch of the blood vessel through the lesion area, and the microguidewire was withdrawn. After confirming the true lumen of the blood vessel, the Transcend 300 microguidewire (Stryker) was inserted to withdraw the microcatheter. Then, the Gateway balloon catheter (Boston Scientific, USA) was sent along the Transcend Floppy 300 guidewire to the distal end of the lesion area, such that the size of the balloon catheter reached $80 \%$ of the lesion stenosis and the balloon was expanded slowly. After satisfactory balloon expansion, it was withdrawn, and the Select Plus microcatheter (Stryker) was inserted along the exchange guidewire. The Enterprise stent (Codman) was delivered to the lesion area through the microcatheter. It was necessary that the stent completely cover the lesion, and the length needed to be greater than $3-5 \mathrm{~mm}$ at both ends of the lesion area. After stenting, angiography was performed to assess the procedure results and rule out thrombosis in the stent or distal thromboembolism. The patient's blood pressure was maintained at $120-140 \mathrm{mmHg}$ after treatment, and the patient continued to receive dual antithrombotic therapy, including clopidogrel 
(75 mg/d) for six weeks and aspirin $(100 \mathrm{mg} / \mathrm{d})$ for at least six months. Patients received follow-up telephone calls 30 days after the procedure.

2.1.3. Data Collection and Outcomes. We reviewed medical records and extracted basic information concerning the patients and lesions, including demographic data, lesion characteristics, clinical presentations, modified Rankin scale (mRS) scores, and the degree of arterial stenosis. Indicators related to stent placement were recorded, including the size of the balloon catheter and Enterprise stent, complications within 30 days after stenting, and postoperative angiography results.

The safety was assessed by noting the occurrence of adverse events within 30 days after stenting, including TIA, stroke, or death, based on the guidelines of the SAMMPRIS trial. Besides, we recorded the rate of technical success, which was defined as remaining stenosis of $50 \%$ or less as measured by immediate postoperative angiography, which indicated the precise release of the Enterprise stent into the lesion area.

\subsection{Literature Review}

2.2.1. Study Protocol and Search Strategy. We conducted a systematic review of relevant literature, following the Preferred Reporting Items for Systematic Reviews and Meta-Analyses (PRISMA) guidelines [22]. The study was registered in the International System of Review Prospective Register (PROSPERO, CRD42020183509). With the assistance of an experienced librarian, we searched PubMed, EMBASE, Web of Science, Cochrane, and Clinical Trials databases. Keywords, including "intracranial arteriosclerosis," "cerebral arterial diseases," "cerebral arteries," "internal carotid," "vertebrobasilar arteries," "middle cerebral artery," "stents," and "Enterprise" were used in both "AND" and "OR" combinations, as described in Table S1. The literature search period was from the earliest date available for each database to May 2020. All studies reporting ICAS patients treated with the Enterprise stent were selected.

2.2.2. Trial Selection. Studies reporting an ICAS case series treated with Enterprise stents with a sample size greater than five met the initial inclusion criteria. We reviewed all potentially qualified studies with results specifically related to safety or effectiveness. We excluded studies that did not provide perioperative complication rates and technical success rates. Duplicate studies were moved. Non-English articles, conference abstracts without full text, and case series combined with complete occlusion of the cerebral artery and moyamoya disease also were excluded.

2.2.3. Data Extraction. Two investigators (BWS and CX) independently extracted the following information from each eligible study: the total number of patients and lesions treated with the Enterprise stent, pretreated and posttreated mean stenosis degree, technical success rate, intraprocedural complications, frequency of stroke, TIA, or death within 30 days after implantation of the Enterprise stent, frequency and mean duration of clinical and imaging follow-ups, frequency of stroke, TIA or death in the territory of the qualifying artery beyond 30 days, and the in-stent restenosis (ISR) and symptomatic ISR rates. The presence of ISR was indicated by a greater than $50 \%$ residual stenosis in the stent after placement as assessed by follow-up DSA or computed tomographic angiography (CTA) examination.

2.2.4. Qualitative Assessment. Two investigators (BWS and $\mathrm{CX}$ ) independently assessed the quality of the included literature, and a third investigator resolved any disagreements. Literature quality was assessed using a modified version of the Newcastle-Ottawa Quality Assessment Scale (NOS) [23], which was specifically designed to assess the quality of nonrandomized studies, such as case-control studies and cohort studies. We evaluated the quality of each study based on three aspects, including (1) selection of the study groups, (2) comparability of the study groups, and (3) achievement of the outcome of interest. Specific evaluation details are shown in Table S2.

2.3. Statistical Analysis. All statistical analyses were performed using R software (version 3.6.1, R Core Team, Vienna, Austria). Standard descriptive statistics were used for the institutional series. Continuous data were presented as means \pm standard deviation. Categorical data were presented as percentages. Since all included studies were noncomparative studies, we calculated incidence rates rather than relative risks or mean differences. The cumulative incidence and 95\% confidence interval (CI) for all events were recorded, and cumulative outcomes were calculated. Subgroup analyses were conducted based on the anterior circulation (AC) and posterior circulation (PC) of the cerebral arteries. For the pooled analysis, event rates were summarized using a random-effects model if heterogeneity was significant; otherwise, a fixed-effects model was used [24]. Study heterogeneity was evaluated using the $I^{2}$ statistic. $I^{2}$ values of $0-25 \%, 26-50 \%, 51-75 \%$, and $>75 \%$ indicated light, low, moderate, and high heterogeneity, respectively [25]. If any apparent heterogeneity was observed, a sensitivity analysis was used to explore the source of the heterogeneity. Visualization using a funnel plot was employed to assess publication bias when there were sufficient numbers of eligible studies to create the plot. Asymmetric funnel plots are suggestive of publication bias.

\section{Results}

\subsection{Institutional Series}

3.1.1. Patient Population and Lesion Characteristics. Three hundred twenty-one ICAS patients received PTAS treatment at our center from June 13, 2017, to April 3, 2020. After excluding ineligible participants, 104 patients (mean age, $58.61 \pm 9.32$ years) with 105 stenosis lesions were incorporated into the present study (60 male patients, $57.69 \%$ ). The screening flowchart is seen in Figure S1. All relevant patient data are shown in Table 1. Forty-seven (44.76\%) lesions were located in the AC (13 in the intracranial segment of the internal carotid artery, ICA (12.38\%); and 34 in the middle cerebral artery, MCA (32.38\%)). Fifty-eight (55.24\%) lesions were located in the PC (15 in the 
TABLE 1: The patient's demographic data, clinical and angiographic outcome.

\begin{tabular}{|c|c|}
\hline Characteristic & Value \\
\hline Age, years $($ mean $\pm S D)$ & $58.61 \pm 9.32$ \\
\hline Sex, male/female $(n)$ & $60 / 44$ \\
\hline \multicolumn{2}{|l|}{ Qualifying event ( $n(\%))$} \\
\hline Transient ischemic attack & $21(20.19)$ \\
\hline Cerebral infarction & $83(79.81)$ \\
\hline \multicolumn{2}{|l|}{ Comorbidities ( $n(\%))$} \\
\hline Hypertension & $80(76.92)$ \\
\hline Diabetes & $30(28.85)$ \\
\hline Coronary artery disease & $18(17.31)$ \\
\hline \multicolumn{2}{|l|}{ Pretreated modified Rankin scale score $(n(\%))$} \\
\hline 0 & $17(16.35)$ \\
\hline 1 & $69(66.35)$ \\
\hline 2 & $16(15.38)$ \\
\hline 3 & $1(0.96)$ \\
\hline \multicolumn{2}{|l|}{ Location $(n(\%))$} \\
\hline Intracranial segment of internal carotid artery & $13(12.38)$ \\
\hline Middle cerebral artery & $34(32.38)$ \\
\hline Basilar artery & $43(40.95)$ \\
\hline Intracranial segment of the vertebral artery & $15(14.28)$ \\
\hline \multicolumn{2}{|l|}{ Lesion morphology $(n(\%))$} \\
\hline A & $14(13.33)$ \\
\hline $\mathrm{B}$ & $40(38.10)$ \\
\hline $\mathrm{C}$ & $51(48.57)$ \\
\hline \multicolumn{2}{|l|}{ Complications ( $n(\%))$} \\
\hline Any stroke or death within $30 \mathrm{~d}$ & $7(6.73)$ \\
\hline Nonfatal ischemic stroke within $30 \mathrm{~d}$ & $4(3.85)$ \\
\hline Nonfatal hemorrhage stroke within $30 \mathrm{~d}$ & $2(1.92)$ \\
\hline Death within $30 \mathrm{~d}$ & $1(0.96)$ \\
\hline \multicolumn{2}{|l|}{ Angiographic outcome } \\
\hline Pretreated stenosis degree (\%) (mean $\pm \mathrm{SD})$ & $87.13 \pm 7.80$ \\
\hline Posttreated stenosis degree $(\%)($ mean \pm SD) & $27.31 \pm 8.89$ \\
\hline Length of stenosis (mean $\pm \mathrm{SD}$ ) & $10.68 \pm 4.99$ \\
\hline Enterprise stent length $(\mathrm{mm})($ mean $\pm \mathrm{SD})$ & $25.12 \pm 5.03$ \\
\hline Balloon catheter length $(\mathrm{mm})($ mean $\pm \mathrm{SD})$ & $13.22 \pm 3.64$ \\
\hline
\end{tabular}

intracranial segment of the vertebral artery, VA (14.28\%); and 43 in the basilar artery, BA (40.95\%)). Twenty-one (20.19\%) patients were admitted to the hospital for TIA and $83(79.81 \%)$ for stroke. The frequency of preoperative mRS scores were as follows: 0, 17 (16.35\%); 1, 69 (66.35\%); 2, $16(15.38 \%)$; and 3, $1(0.96 \%)$. The preoperative degree of arterial stenosis was $87.13 \pm 7.80 \%$, as determined by DSA.

3.1.2. Immediate Angiographic and 30-Day Outcomes. All 105 stents met the technical success criteria, resulting in a $100 \%$ success rate, and postoperative stenosis averaged $27.31 \pm 8.89 \%$ (Table 1 ). Within 30 days after stent placement, 7 (6.73\%) patients developed stroke or died, 4 (3.81\%) patients experienced an ischemic stroke, and 3
(2.88\%) patients developed a hemorrhagic stroke. One patient with a hemorrhagic stroke died, yielding a total mortality rate of $0.95 \%$. One patient with a right MCA stenosis experienced hyperperfusion cerebral hemorrhage on the second day after stent placement and died, despite immediate symptomatic treatment. One patient with stenosis at the end of the left ICA developed a subarachnoid hemorrhage 12 hours after the stent was implanted. This patient subsequently underwent decompressive craniectomy, gradually achieved full recovery, and exhibited a mRS score of 3 on day 30 after the procedure. One patient with cerebellar hemorrhage did not present any visible symptoms but scored 1 on the mRS on day 30 following the procedure. Three patients with BA stenosis and one patient with MCA stenosis developed a perforating infarction within 30 days after the procedure, but the symptoms were not severe. After receiving antiplatelet therapy, their symptoms improved, and their mRS scores were 0-2 at the 30-day telephone follow-up interview.

\subsection{Systematical Review}

3.2.1. Search Results. The literature selection process is shown in Figure 1. The initial database search identified 351 citations. Fifty-two duplicates were excluded, and 291 articles were excluded after reading the titles and abstracts, leaving eight articles. Two articles presented overlapping data, and we chose to include the article with the longest study duration and the largest number of cases. One additional study was excluded for including only patients with complete occlusion of the cerebral artery. Therefore, seven studies, including the institutional series, were included in the systematic review.

3.2.2. Characteristics of Included Studies. The baseline information for all studies is shown in Table 2. Studies were published between 2012 and 2019. All included studies were retrospective observational case studies that lacked comparisons to other treatments as control groups. Due to these characteristics, all selected studies had a high risk of bias, as assessed by the NOS scale (Table S2). Five studies were conducted in East Asia, while the other two studies were conducted in Germany and Turkey.

A total of 557 patients underwent Enterprise stent implantation for 588 ICAS lesions. The average age ranged from 56.8 to 64.0 , and the pretreatment mean stenosis ranged from $65.4 \%$ to $92.0 \%$. All studies reported some within 30 days after Enterprise implantation. Five studies, including 343 lesions, reported angiographic follow-up examinations, with the mean time ranging from 6 to 22 months. Five studies, including 370 lesions, reported results from clinical follow-up examinations, with the mean time ranging from 6.2 to 25.6 months.

3.2.3. Immediate Angiographic and 30-Day Outcomes. The summary of adverse events after Enterprise implantation is shown in Table 3, and the forest diagram of the results is shown in Figure 2. The technical success rate ranged from $98.5 \%$ to $100 \%$, with only one procedure that did not achieve technical success. Posttreatment stenosis was reported for 


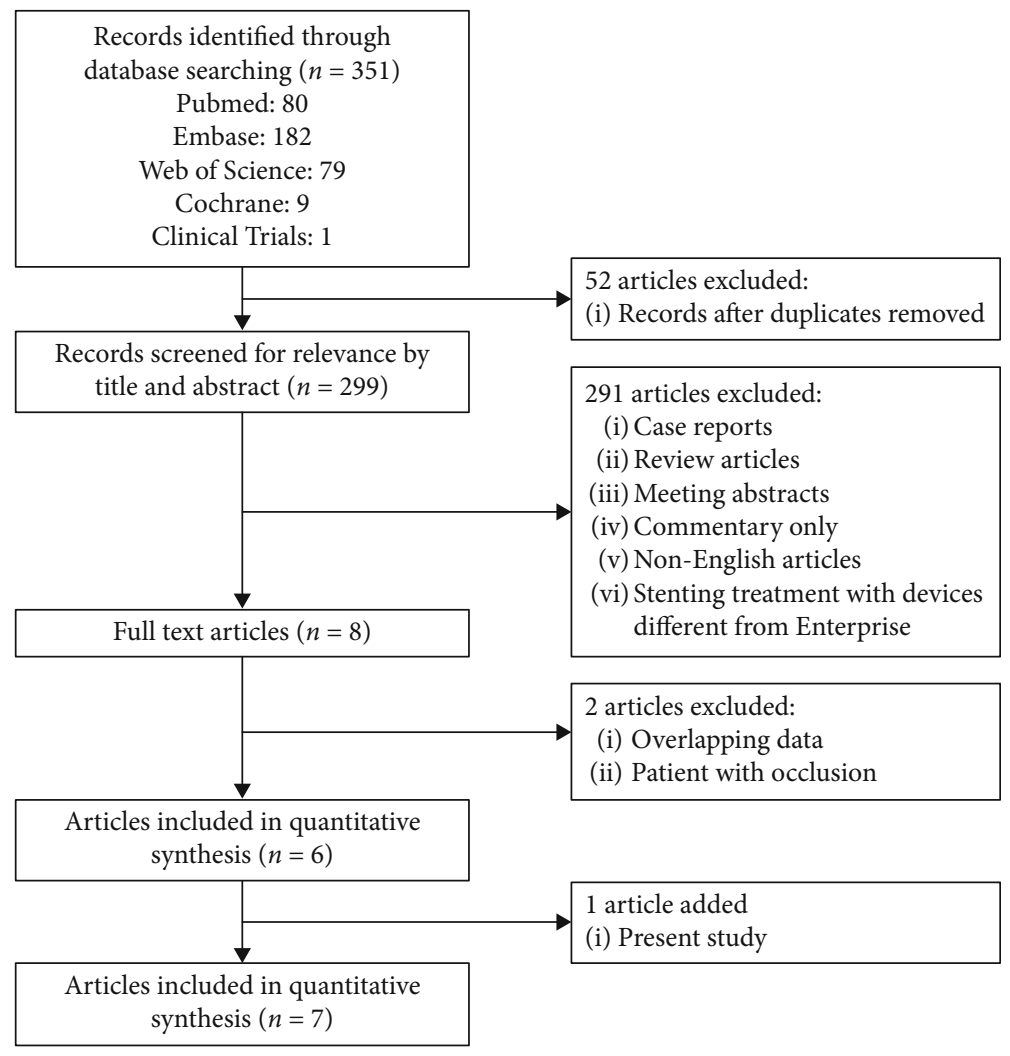

FIGURE 1: Flowchart shows study selection procedure. 7 studies were included in this systematic review.

474 lesions in five studies, ranging from $12 \pm 10 \%$ to 27.31 $\pm 8.89 \%$ (Table 2). Within 30 days following PTAS, the pooled incidence of adverse events was as follows: stroke or death, 7.4\% (95\% CI 5.5\%-10.1\%); hemorrhagic stroke, $3.1 \%$ (95\% CI, $1.9 \%-5.0 \%)$; ischemic stroke, $4.5 \%$ (95\% CI, $3.0 \%-6.73 \%$ ); and mortality, $1.2 \%$ (95\% CI, $0.5 \%-2.6 \%)$. The pooled incidence of intraprocedural complications was $2.2 \%$ (95\% CI, 1.2\%-4.0\%), including vasospasm, hematoma in the groin, and asymptomatic dissection of the stented segment. These results were not heterogeneous $\left(I^{2}=0\right)$, and no apparent publication bias was observed in the funnel chart (Figure S2). We conducted a subgroup analysis of complications that occurred within 30 days by separating patients with lesions into AC and PC subgroups. The complication rate of patients with $\mathrm{AC}$ lesions was $6.7 \%$ (95\% CI, 3.6\%-12.3\%), and for patients with PC lesions was $8.1 \%$ (95\% CI, 4.9\%-13.4\%). There was no significant statistical difference between the two subgroups (Figure S3).

3.2.4. Imaging and Clinical Follow-Up. Five studies, including 370 lesions, reported outcomes observed at clinical follow-up examinations. The pooled incidence of ischemic stroke or TIA in the territory of the qualifying artery beyond 30 days was $3.2 \%(95 \% \mathrm{CI}, 1.1 \%-9.5 \%)$ (Figure 2$)$. Since $I^{2}=56$, we chose the analysis result obtained from the random-effects model. Through sensitivity analysis, we determined that the heterogeneity primarily resulted from the study by Wang et al. [15]. No deaths were reported during the follow-up examinations. Five studies, including 343 lesions, reported imaging follow-up results. The pooled incidence of ISR was $10.1 \%$ (95\% CI, $4.6 \%-22.2 \%$ ), and the pooled incidence of symptomatic ISR was $4.9 \%$ (95\% CI, 2.9\%-8.5\%). The results of ISR were highly heterogeneous $\left(I^{2}=75\right)$. Using sensitivity analysis, we established that the heterogeneity principally resulted from the study by Vajda et al. [17]. We speculated that the heterogeneity might be caused by differences in the length of follow-up times. Based on the asymmetry of the funnel chart, we believe that the three results described above presented some degree of publication bias (Figure S2).

\section{Discussion}

We summarized our experience in a high-volume center and all published studies before May 2020 on the treatment of ICAS with the Enterprise stent and evaluated the safety and efficacy of the Enterprise stent. An analysis of 588 lesions in 557 patients revealed that the incidence of stroke or mortality within 30 days after the procedure was 7.4\% (95\% CI 5.5\%$10.1 \%)$, and all but one procedure obtained technical success. In the SAMMPRIS and VISSIT trials, the incidence of adverse events within 30 days of PTAS with non-Enterprise stents was $14.7 \%$ and $24.1 \%$, respectively, which are higher than the rates reported in this study. The long-term effect of treatment, as assessed by the incidence of ischemic stroke or TIA in the territory of the qualifying artery beyond 30 days, was 3.2\% (95\% CI, 1.1\%-9.5\%). Thus, our findings provided evidence to support the safety and effectiveness of Enterprise stent placement in the treatment of ICAS. 


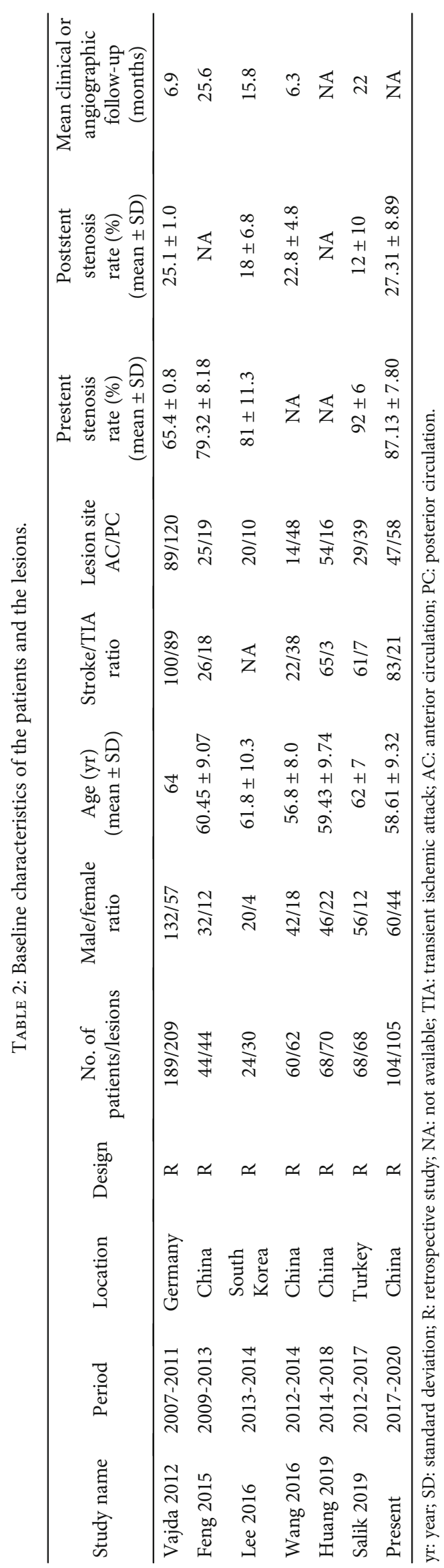


TABLE 3: Summary of adverse events after Enterprise implantation.

\begin{tabular}{|c|c|c|c|c|c|c|c|c|c|}
\hline \multirow{3}{*}{ Study } & \multirow{3}{*}{$\begin{array}{l}\text { Intraprocedural } \\
\text { complications }\end{array}$} & \multicolumn{4}{|c|}{ Any stroke or death within 30 days } & \multirow{3}{*}{$\begin{array}{l}\text { Ischemic stroke or } \\
\text { TIA in the } \\
\text { territory of the } \\
\text { qualifying artery } \\
\text { beyond } 30 \text { days }\end{array}$} & \multirow{3}{*}{$\begin{array}{l}\text { Mortality } \\
\text { beyond } \\
30 \text { days }\end{array}$} & \multicolumn{2}{|c|}{ ISR } \\
\hline & & Stoke & & Nonfatal & Death & & & & \\
\hline & & Hemorrhagic & Ischemic & & & & & 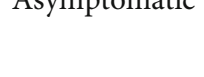 & symptomiatic \\
\hline Vajda 2012 & $4 / 209$ & $8 / 189$ & $10 / 189$ & $16 / 189$ & $2 / 189$ & 4 & $0 / 174$ & $39 / 174$ & $4 / 44$ \\
\hline Feng 2015 & $0 / 44$ & $1 / 44$ & $3 / 44$ & $4 / 44$ & $0 / 44$ & 0 & $0 / 44$ & $1 / 44$ & $2 / 44$ \\
\hline Lee 2016 & $0 / 30$ & $1 / 24$ & $2 / 24$ & $2 / 24$ & $1 / 24$ & 0 & $0 / 24$ & $1 / 20$ & $0 / 20$ \\
\hline Wang 2016 & $3 / 62$ & $0 / 60$ & $2 / 60$ & $2 / 60$ & $0 / 60$ & 5 & $0 / 60$ & $1 / 45$ & $5 / 45$ \\
\hline Huang 2019 & $0 / 70$ & $2 / 68$ & $1 / 68$ & $3 / 68$ & $0 / 68$ & NA & NA & NA & NA \\
\hline Salik 2019 & $1 / 68$ & $0 / 68$ & $0 / 68$ & $1 / 68$ & $0 / 68$ & 0 & $0 / 68$ & $2 / 60$ & $0 / 60$ \\
\hline Present & $0 / 105$ & $3 / 104$ & $4 / 104$ & $6 / 104$ & $1 / 104$ & NA & NA & NA & NA \\
\hline
\end{tabular}

NA: not available.

The endovascular procedure emerged as a novel ICAS treatment in the 1980s. Although technology and equipment have undergone constant innovation and improvement, the procedure has never become the predominant treatment for ICAS $[26,27]$. Results of the WAISD and SAMMPRIS trials confirmed the safety of AMT [5]. However, for patients with high-grade stenosis, the rate of stroke recurrence after AMT was close to $20 \%$ per year [11]. For many patients with severe ICAS (stricture $>70 \%$ ), PTAS treatment is still an important alternative treatment. Additionally, the results of the SAMMPRIS trial have been criticized by some experts due to limitations in stent selection, patient inclusion, and technical aspects of the procedures [28]. Therefore, exploring optimal treatments for ICAS is still a worthy endeavor.

After minimizing the limitations of the SAMMPRIS trial, an RCT in China, China Angioplasty and Stenting for Symptomatic Intracranial Severe Stenosis (CASSISS) [29], was initiated and is ongoing. Early reports from the CASSISS trial differed from the SAMMPRIS study results, including the observation that the incidence of 30-day adverse events in patients with high-grade ICAS treated by PTAS was only $4.3 \%$. This result increased the confidence of practitioners to use PTAS for ICAS treatment in China. The extensive use of Wingspan stents in the SAMMPRIS trial also has been widely criticized [12]. Some experts believed that the rigidity and high radial force of the Wingspan stent were related to the high incidence of perioperative complications [30]. Recently, the Wingspan Stent System Post Market Surveillance Study (WEAVE) trial [31] and the Wingspan Oneyear Vascular Events and Neurologic Outcomes (WOVEN) trial [32] reported acceptable results. As postmarket surveillance studies, the WEAVE and WOVEN trials strictly enrolled patients treated on-label with the Wingspan stent and reported that $2.67 \%$ of patients had died or developed stroke within 72 hours, and $8.5 \%$ of patients had died or developed stroke at the one-year follow-up. These results suggest that when assessing the use of PTAS for ICAS treatment, the choice of an appropriate patient group is critical.

Following the failure of the SAMMPRIS and VISSIT trials, studies on the feasibility and effectiveness of using other alternative stents to treat symptomatic ICAS have been continuing $[9,13-20]$. Among the many stent options, the self-expanding Enterprise stent has been used frequently for ICAS treatment, especially in our center. The Enterprise stent, which was specifically developed to treat wide-necked intracranial cerebral aneurysms, has a closed-cell design, special carrier system, and lower radial force compared with the Wingspan stent [15]. It has a diameter of $4.5 \mathrm{~mm}$ and has four lengths of $14,22,28$, and $37 \mathrm{~mm}$, so it is suitable for intracranial blood vessels with a diameter of $2.5-4.0 \mathrm{~mm}$. The release rate of the Enterprise stent is less than 70\%, and it is recyclable. More importantly, the Enterprise stent is exceptionally malleable, and its delivery catheter tip is soft and flexible, making it easier to reach the lesion area than more rigid stents [13]. Due to the wide application of the Enterprise stent to treat aneurysm embolization [33], it has been reported that it could reach areas inaccessible by other types of stents, such as the Neuroform EZ and Solitaire stents [34]. Interestingly, Vajda et al. proposed that the Enterprise stent could be delivered to any part of the circle of Willis with the aid of microcatheters [13].

Several previous studies reported low perioperative complication rates when using the Enterprise stent to treat ICAS that ranged from $1.47 \%$ to $12.50 \%[9,13-17]$. The results of this study agree with previous reports, as only $7.4 \%$ (95\% CI $5.5 \%-10.1 \%$ ) of patients experienced stroke or death within 30 days after Enterprise stent implantation. More adverse events were caused by ischemic events, 4.5\% (95\% CI $3.0 \%-6.7 \%$ ), and although the incidence of hemorrhagic events was slightly lower, 3.1\% (95\% CI 1.9\%-5.0\%), death was always related to hemorrhagic stroke. Additionally, we conducted a subgroup analysis based on the AC/PC lesion location, and the complication rate was not significantly different between the two subgroups.

Several studies reported additional complications related to the procedure, such as vasospasm and stent migration [9, 13]. The periprocedural complication rates of the current study were undoubtedly better than the stent group of the SAMMPRIS trial but slightly higher than the WEAVE trial, the early results of the CASSISS trial, and the AMT group of the SAMMPRIS trial. These results could be related to patient selection, operator experience, and characteristics of individual PTAS. As the complication rate within 30 days after PTAS in the VISSIT trial was as high as $24.1 \%$, the trial 


\begin{tabular}{|c|c|c|c|c|c|c|c|}
\hline Study & Events & Total & & Proportion & $95 \%-C I$ & $\begin{array}{l}\text { Weight } \\
\text { (fixed) }\end{array}$ & $\begin{array}{c}\text { Weight } \\
\text { (random) }\end{array}$ \\
\hline Salik2019 & 1 & 68 & +1 & 0.015 & {$[0.000 ; 0.079]$} & $2.5 \%$ & $2.8 \%$ \\
\hline Huang2019 & 3 & 68 & & 0.044 & {$[0.009 ; 0.124]$} & $7.6 \%$ & $8.5 \%$ \\
\hline Wang2016 & 2 & 60 & -7 & 0.033 & {$[0.004 ; 0.115]$} & $5.0 \%$ & $5.7 \%$ \\
\hline Lee2015 & 3 & 24 & & 0.125 & {$[0.027 ; 0.324]$} & $8.3 \%$ & $9.3 \%$ \\
\hline Feng2015 & 4 & 44 & + & 0.091 & {$[0.025 ; 0.217]$} & $10.7 \%$ & $11.7 \%$ \\
\hline Vajda2012 & 18 & 209 & $-1+$ & 0.086 & {$[0.052 ; 0.133]$} & $47.7 \%$ & $42.9 \%$ \\
\hline Present & 7 & 104 & - & 0.067 & {$[0.027 ; 0.134]$} & $18.2 \%$ & $19.1 \%$ \\
\hline \multirow{2}{*}{\multicolumn{2}{|c|}{$\begin{array}{l}\text { Fixed effect model } \\
\text { Random effect model }\end{array}$}} & 577 & & 0.074 & {$[0.055 ; 0.101]$} & $100.0 \%$ & -- \\
\hline \multirow{2}{*}{\multicolumn{3}{|c|}{$\begin{array}{l}\text { Random effect model } \\
\text { Heterogeneity: } I^{2}=7 \%, \tau^{2}=0.0155, p=0.37\end{array}$}} & & 0.073 & {$[0.052 ; 0.101]$} & -- & $100.0 \%$ \\
\hline & & & $\begin{array}{lllllll} & 0.05 & 0.1 & 0.15 & 0.2 & 0.25 & 0.3\end{array}$ & & & & \\
\hline
\end{tabular}

(a)

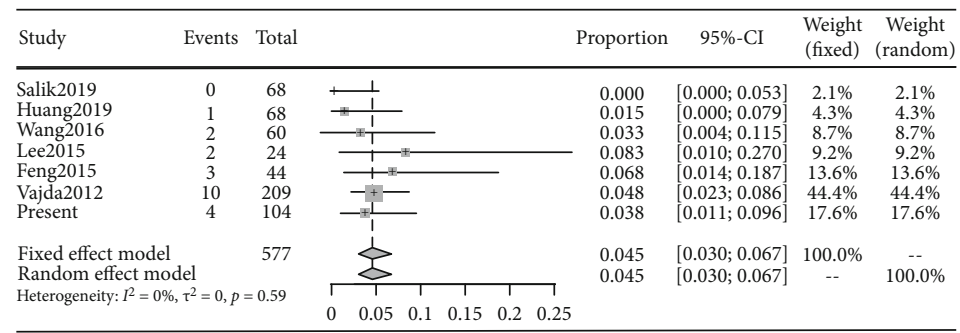

(b)

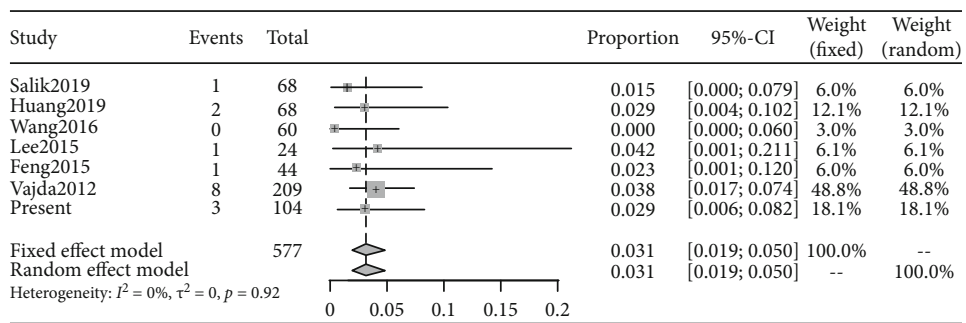

(c)

\begin{tabular}{|c|c|c|c|c|c|c|c|c|c|c|}
\hline Study & Events & Total & & & & & Proportion & 95\%-CI & $\begin{array}{l}\text { Weight } \\
\text { (fixed) }\end{array}$ & $\begin{array}{c}\text { Weight } \\
\text { (random) }\end{array}$ \\
\hline Salik2019 & 0 & 68 & \pm 1 & & & & 0.000 & {$[0.000 ; 0.053]$} & $8.3 \%$ & $8.3 \%$ \\
\hline Huang2019 & 0 & 68 & $\pm \frac{1}{4}$ & & & & 0.000 & {$[0.000 ; 0.053]$} & $8.3 \%$ & $8.3 \%$ \\
\hline Wang2016 & 0 & 60 & $=1$ & & & & 0.000 & {$[0.000 ; 0.060]$} & $8.3 \%$ & $8.3 \%$ \\
\hline Lee2015 & 1 & 24 & $T^{+}$ & & & & 0.042 & {$[0.001 ; 0.211]$} & $17.1 \%$ & $17.1 \%$ \\
\hline Feng2015 & 0 & 44 & $=1$ & & & & 0.000 & {$[0.000 ; 0.080]$} & $8.3 \%$ & $8.3 \%$ \\
\hline Vajda2012 & 2 & 209 & ++ & & & & 0.010 & {$[0.001 ; 0.034]$} & $33.2 \%$ & $33.2 \%$ \\
\hline Present & 1 & 104 & -7 & & & & 0.010 & {$[0.000 ; 0.052]$} & $16.6 \%$ & $16.6 \%$ \\
\hline \multirow{3}{*}{\multicolumn{3}{|c|}{$\begin{array}{l}\text { Fixed effect model } \\
\text { Random effect model } \\
\text { Heterogeneity: } I^{2}=0 \%, \tau^{2}=0, p=0.91\end{array}$}} & 8 & & & & \multirow{3}{*}{$\begin{array}{l}0.012 \\
0.012\end{array}$} & \multirow{3}{*}{$\begin{array}{l}{[0.005 ; 0.026]} \\
{[0.005 ; 0.026]}\end{array}$} & \multirow{3}{*}{$\begin{array}{c}100.0 \% \\
--\end{array}$} & \multirow{3}{*}{$100.0 \%$} \\
\hline & & & & & & & & & & \\
\hline & & & 0.05 & 0.1 & 0.15 & 0.2 & & & & \\
\hline
\end{tabular}

(d)

\begin{tabular}{|c|c|c|c|c|c|c|c|}
\hline Study & Events & Total & & Proportion & $95 \%$-CI & $\begin{array}{l}\text { Weight } \\
\text { (fixed) }\end{array}$ & $\begin{array}{c}\text { Weight } \\
\text { (random) }\end{array}$ \\
\hline Salik2019 & 1 & 68 & $\rightarrow 1$ & 0.015 & {$[0.000 ; 0.079]$} & $9.9 \%$ & $9.9 \%$ \\
\hline Huang2019 & 0 & 68 & 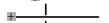 & & $0.000 \cdot 0.053$ & $4.9 \%$ & $4.9 \%$ \\
\hline & 3 & $\begin{array}{l}00 \\
60\end{array}$ & 1 & 0.050 & $0.010 ; 0.139]$ & $30.7 \%$ & $30.7 \%$ \\
\hline Lee2015 & $\begin{array}{l}3 \\
0\end{array}$ & $\begin{array}{l}00 \\
24\end{array}$ & & $\begin{array}{l}0.050 \\
0.000\end{array}$ & {$[0.000 ; 0.142]$} & $\begin{array}{l}5.7 \% \\
5.0 \%\end{array}$ & $\begin{array}{l}5.0 \% \\
5.0 \%\end{array}$ \\
\hline Feng2015 & 0 & 44 & $=1$ & 0.000 & {$[0.000 ; 0.080]$} & $4.9 \%$ & $4.9 \%$ \\
\hline Vajda2012 & 4 & $\begin{array}{l}44 \\
209\end{array}$ & & 0.019 & {$[0.005 ; 0.048]$} & $39.7 \%$ & $39.7 \%$ \\
\hline Present & 0 & 104 & \pm-1 & 0.000 & {$[0.000 ; 0.035]$} & $4.9 \%$ & $4.9 \%$ \\
\hline \multirow{2}{*}{$\begin{array}{l}\text { Fixed effect model } \\
\text { Random effect mode } \\
\text { Heterogeneity: } I^{2}=0 \% \text {, }\end{array}$} & & 577 & $\dot{8}$ & $\begin{array}{l}0.022 \\
0.022\end{array}$ & {$[0.012 ; 0.040]$} & $100.0 \%$ & $-\overline{-}$ \\
\hline & $=0, p=0$. & & 1 & & & & \\
\hline
\end{tabular}

(e)

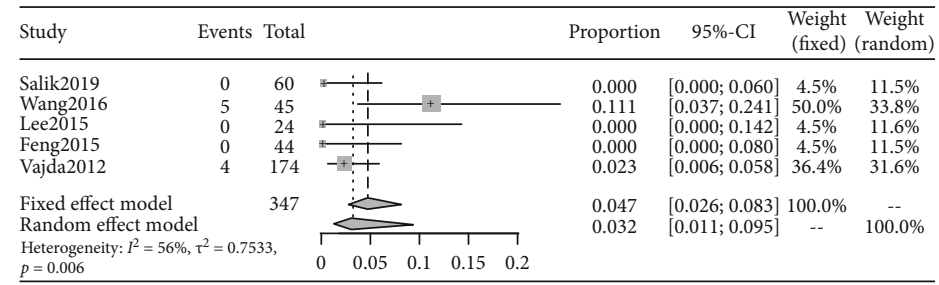

(f)

FIgURE 2: Continued. 


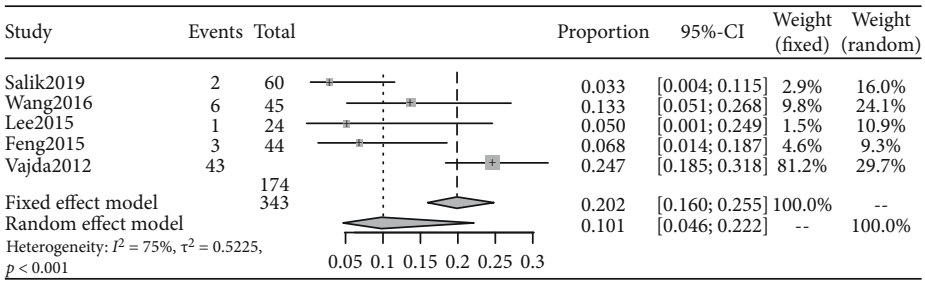

(g)

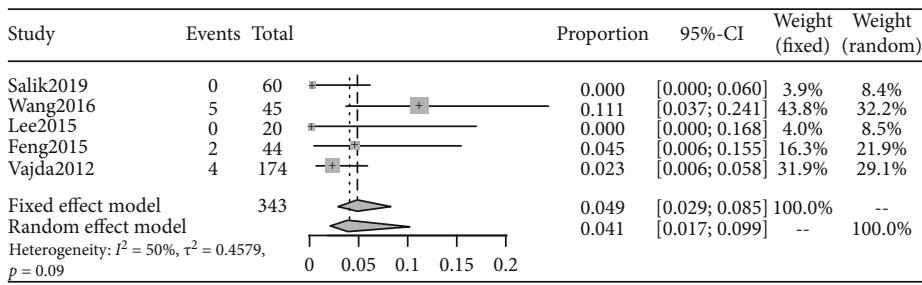

(h)

Figure 2: Pooled analysis outcome. (a) Any stroke or death within 30 days. (b) Ischemic stroke within 30 days. (c) Hemorrhage stroke within 30 days. (d) Mortality within 30 days. (e) Intraprocedure complication. (f) Ischemic stroke or TIA in the territory of the qualifying artery beyond 30 days. (g) In-stent restenosis during imaging follow-up. (h) Symptomatic in-stent restenosis during imaging follow-up.

was terminated early. It is generally considered that the balloon-expandable stent is more rigid and less flexible than self-expanding stents and may be difficult to navigate along curved blood vessels [24]. Some experts believed that PTAS complications were related to the morphological classification of lesions, as proposed by Mori et al. [9, 14, 35], and the complication rates of type $\mathrm{B}$ and type $\mathrm{C}$ lesions were higher. Recently, a multicenter, single-arm study involving 159 patients explored the application of balloon-expandable stents in the treatment of ICAS. The complication rate at 72 hours after surgery was $0 \%$, but the study included more Mori A (33.3\%) and Mori B (52.2\%) lesions, so the therapeutic effect of balloon-expandable stents for Mori C lesions is still worth exploring [36].

There are relatively few studies on other types of stents, so it is challenging to draw broad conclusions [18, 19]. A single-center study involving 76 patients explored the effect of a new generation of closed-cell self-expandable stents, the Acclino ${ }^{\circledR}$ flex stent, in the treatment of ICAS. The incidence of stroke or death within 30 days after PTAS was $6.5 \%$, which is similar to the results of this study [19]. Also, another study reported that using the Neuroform EZ stent to treat ICAS has the possibility of reducing complication risks. That study included 71 consecutive patients, and no stroke or death was observed within 30 days after surgery. The open-cell design of the Neuroform EZ stent was thought to be associated with this result [18]. However, the exact risk factors for these perioperative complications after PTAS with the Enterprise stent are still uncertain, and further research is needed [37]. In particular, for high-grade stenosis, the low complication rate in our study supported the safety of Enterprise stent implantation in ICAS treatment.

For the follow-up results of the systematic review, the incidence of stroke, TIA, or death over 30 days after PTAS was $3.2 \%$ (95\% CI, 1.1\%-9.5\%). This result was better than the AMT (6.4\%) and stent groups (5.3\%) in the SAMMPRIS trial and lower than the AMT (5.7\%) and stent groups
$(12.1 \%)$ in the VISSIT trial. These results indicated that the long-term stroke prevention of the Enterprise stent was relatively good. Long-term complications are often associated with ISR, and the high incidence of ISR after stent implantation for ICAS has long been a major disadvantage of placing stents [38]. ISR is common in the first year after PTAS and an important cause of nonsurgical ischemic events after stent placement [10]. Importantly, we found that the ISR rate during the follow-up period after Enterprise stent implantation was $10.1 \%$ (95\% CI 4.6\%-22.2\%), and the incidence of symptomatic ISR was $4.9 \%$ (95\% CI $2.9 \%-8.5 \%)$. These results are in line with results from a previous metaanalysis of PTAS for ICAS treatment [39]. The Wingspan stent had an ISR rate between $6 \%$ and $42.8 \%$ [9], and the ISR rate was as high as $26.5 \%$ in the VISSIT trial using the balloon-expandable stent. The ISR rate in the study by Vajda et al. was $24.71 \%$, which potentially could be related to the patient inclusion criteria and follow-up times, as numerous patients with a stenosis rate of 50\%-70\% were included and followed for a long time until ISR was detected [17].

Recently, a meta-analysis demonstrated that drug-eluting stents performed better in preventing ISR, with an ISR incidence of only $4.1 \%$ and an asymptomatic ISR incidence of $3.0 \%$ [20]. However, the risk of low-grade chronic inflammation leading to late stent thrombosis limits the application of drug-eluting stents. Moreover, drug-eluting stents are quite stiff, making it challenging to reach complex stenosis lesions, causing drug-eluting stents to be inferior to the Enterprise stent in terms of operability [20].

Several relevant studies have indicated that ISR may be related to lesion location, preoperative stenosis, use of a balloon, and the application of antiplatelet drugs [38]. However, few studies have focused on the mechanisms underlying ISR. A few studies have reported that the low radial force of Enterprise stents might play a role in reducing the ISR rate because it is related to intimal hyperplasia [33]. Currently, treatment for ISR includes medical therapy, 
bypass surgery, and endovascular recanalization, but most of these treatments have unsatisfactory outcomes [38]. In our center, the use of antiplatelet agents was strictly regulated based on the WASID trial to better prevent the emergence of ISR.

\section{Limitations}

Limitations associated with a retrospective single-arm study were unavoidable in the institutional series. Despite the authenticity of medical records, recall bias and selection bias were common, and the lack of a control group limited the scope of the results. For the systematic review, all included studies were retrospective single-center studies and lacked appropriate control groups for comparison with other treatments. The follow-up times and imaging methods for each study were highly variable, which may have confounded the clinical and angiographic results. The small number of available studies limited the effectiveness of evaluating publication bias. Also, this study only included relevant studies that took place in the last ten years. Over time, stenting technologies have continuously improved. The complication rate has decreased, which might be partially responsible for the heterogeneity in the results when studies published across a long-time span were compared. According to the Grading of Recommendations, Assessment, Development, and Evaluation framework, we found that the heterogeneity and methodological limitations of the included studies negatively impacted data in this study [40-42]. Nevertheless, with 557 patients and 588 lesions, this systematic review was the most extensive study to date that investigated the use of the Enterprise stent for ICAS. This study provided useful data to evaluate the effects of the Enterprise stent in the treatment of ICAS.

\section{Conclusion}

The use of the Enterprise stent for intracranial angioplasty may be safe and effective in the treatment of ICAS. When used appropriately, the vast majority of ICAS patients receiving Enterprise stent implantation obtained good outcomes and excellent neurological performance during the followup period. However, considering the limitations associated with the level of evidence in this study, additional RCTs are needed to further verify the effects of Enterprise stents for ICAS. Furthermore, the results of this study might provide assistance in the selection of stents for endovascular treatment of symptomatic severe ICAS.

\section{Data Availability}

The statistical results of our single center, as well as the data of the system review and the results of the pooled analysis, can be obtained in the manuscript and supplementary materials.

\section{Conflicts of Interest}

The authors declare that there is no conflict of interest regarding the publication of this paper.

\section{Authors' Contributions}

BWS and CX have contributed equally to this paper. BWS did the search, screening, and quality assessment of the articles, coding articles, conducting the analysis, and writing and revising the manuscript. CX did the search, screening, and quality assessment of the articles, coding articles, conducting the analysis, and writing and revising the manuscript. PW monitored the data collection and analyzed the data. ML monitored the data collection and analyzed the data. SCX conducted the study of single-center case series and collected and analyzed the data. CLW conducted the study of singlecenter case series and collected and analyzed the data. XYL did the statistical analysis and editing of the manuscript. YPL did the statistical analysis and editing of the manuscript. HZS did the study designer, overall methodological supervision, checking the analysis, and main manuscript reviewer.

\section{Acknowledgments}

We thank EditSprings for the English revision. This study was funded by the National Nature Science Foundation of China (Grant nos. 82071309), Natural Science Foundation of Heilongjiang Province of China (Grant nos. YQ2019H015), and Natural Science Foundation of Heilongjiang Province of China (Grant nos. ZD2018018).

\section{Supplementary Materials}

Table S1: database search strategy. Table S2: a modified version of the Newcastle-Ottawa Quality Assessment Scale. Figure S1: screening flowchart of the institutional series. Figure S2: funnel chart for detecting publication bias. Figure S3: subgroup analysis based on the anterior and posterior circulation of cerebral artery. (Supplementary Materials)

\section{References}

[1] C. Banerjee and M. I. Chimowitz, "Stroke caused by atherosclerosis of the major intracranial arteries," Circulation Research, vol. 120, no. 3, pp. 502-513, 2017.

[2] R. V. Krishnamurthi, V. L. Feigin, M. H. Forouzanfar et al., "Global and regional burden of first-ever ischaemic and haemorrhagic stroke during 1990-2010: findings from the Global Burden of Disease Study 2010," The Lancet Global Health, vol. 1, no. 5, pp. e259-e281, 2013.

[3] Y. Wang, X. Zhao, L. Liu et al., "Prevalence and outcomes of symptomatic intracranial large artery stenoses and occlusions in China: the Chinese Intracranial Atherosclerosis (CICAS) Study," Stroke, vol. 45, no. 3, pp. 663-669, 2014.

[4] W. N. Kernan, B. Ovbiagele, H. R. Black et al., "Guidelines for the prevention of stroke in patients with stroke and transient ischemic attack," Stroke, vol. 45, no. 7, pp. 2160-2236, 2014.

[5] M. I. Chimowitz, M. J. Lynn, H. Howlett-Smith et al., "Comparison of warfarin and aspirin for symptomatic intracranial arterial stenosis," The New England Journal of Medicine, vol. 352, no. 13, pp. 1305-1316, 2005.

[6] A. Wabnitz and M. Chimowitz, "Angioplasty, stenting and other potential treatments of atherosclerotic stenosis of the intracranial arteries: past, present and future," Stroke, vol. 19, no. 3, pp. 271-276, 2017. 
[7] C. P. Derdeyn, M. I. Chimowitz, M. J. Lynn et al., "Aggressive medical treatment with or without stenting in high-risk patients with intracranial artery stenosis (SAMMPRIS): the final results of a randomised trial," Lancet, vol. 383, no. 9914, pp. 333-341, 2014.

[8] O. O. Zaidat, B. F. Fitzsimmons, B. K. Woodward et al., "Effect of a balloon-expandable intracranial stent vs medical therapy on risk of stroke in patients with symptomatic intracranial stenosis: the VISSIT randomized clinical trial," Journal of the American Medical Association, vol. 313, no. 12, pp. 12401248, 2015.

[9] Z. Feng, G. Duan, P. Zhang et al., "Enterprise stent for the treatment of symptomatic intracranial atherosclerotic stenosis: an initial experience of 44 patients," BMC Neurology, vol. 15, no. 1, p. 187, 2015.

[10] C. P. Derdeyn, D. Fiorella, M. J. Lynn et al., "Nonprocedural symptomatic infarction and in-stent restenosis after intracranial angioplasty and stenting in the SAMMPRIS trial (stenting and aggressive medical management for the prevention of recurrent stroke in intracranial stenosis)," Stroke, vol. 48, no. 6, pp. 1501-1506, 2017.

[11] P. Gao, D. Wang, Z. Zhao et al., "Multicenter prospective trial of stent placement in patients with symptomatic high-grade intracranial stenosis," AJNR. American Journal of Neuroradiology, vol. 37, no. 7, pp. 1275-1280, 2016.

[12] M. Fujimoto, Y. Shobayashi, K. Takemoto, S. Tateshima, and F. Viñuela, "Structural analysis for Wingspan stent in a perforator model," Interventional Neuroradiology, vol. 19, no. 3, pp. 271-275, 2013.

[13] A. E. Salik, H. H. Selcuk, H. Zalov, F. Kilinc, M. Cirak, and B. Kara, "Medium-term results of undersized angioplasty and stenting for symptomatic high-grade intracranial atherosclerotic stenosis with Enterprise," Interventional Neuroradiology, vol. 25, no. 5, pp. 484-490, 2019.

[14] C. M. Huang, Y. F. Hong, S. H. Xing et al., “Thirty-day outcomes of the Enterprise stent in treating hypoperfusion of symptomatic intracranial stenosis," World Neurosurgery, vol. 129, pp. e429-e435, 2019.

[15] X. Wang, Z. Wang, C. Wang, Y. Ji, X. Ding, and Y. Zang, "Application of the Enterprise stent in atherosclerotic intracranial arterial stenosis: a series of 60 cases," Turkish Neurosurgery, vol. 26, no. 1, pp. 69-76, 2016.

[16] K. Y. Lee, D. Y. Chen, H. L. Hsu, C. J. Chen, and Y. C. Tseng, "Undersized angioplasty and stenting of symptomatic intracranial tight stenosis with Enterprise: evaluation of clinical and vascular outcome," Interventional Neuroradiology, vol. 22, no. 2, pp. 187-195, 2016.

[17] Z. Vajda, E. Schmid, T. Güthe et al., "The modified Bose method for the endovascular treatment of intracranial atherosclerotic arterial stenoses using the Enterprise stent," Neurosurgery, vol. 70, no. 1, pp. 91-101, 2012.

[18] H. Xu, T. Quan, O. O. Zaidat et al., "Neuroform EZ stenting for symptomatic intracranial artery stenosis: 30 days outcomes in a high-volume stroke center," Frontiers in Neurology, vol. 10, p. 428, 2019.

[19] L. Meyer, H. Leischner, G. Thomalla et al., "Stenting with Acclino ${ }^{\circledR}$ (flex) for symptomatic intracranial stenosis as secondary stroke prevention," Journal of Neurointerventional Surgery, vol. 12, no. 11, pp. 1127-1131, 2020.

[20] G. Ye, X. Yin, X. Yang et al., "Efficacy and safety of drugeluting stent for the intracranial atherosclerotic disease: a systematic review and meta-analysis," Journal of Clinical Neuroscience, vol. 59, pp. 112-118, 2019.

[21] R. A. Agha, C. Sohrabi, G. Mathew et al., "The PROCESS 2020 guideline: updating consensus Preferred Reporting of CasE series in surgery (PROCESS) guidelines," International Journal of Surgery, vol. 84, pp. 231-235, 2020.

[22] D. Moher, A. Liberati, J. Tetzlaff, D. G. Altman, and for the PRISMA Group, "Preferred reporting items for systematic reviews and meta-analyses: the PRISMA statement," $B M J$, vol. 339, no. jul21 1, article b2535, 2009.

[23] A. Stang, "Critical evaluation of the Newcastle-Ottawa scale for the assessment of the quality of nonrandomized studies in meta-analyses," European Journal of Epidemiology, vol. 25, no. 9, pp. 603-605, 2010.

[24] R. Der Simonian and N. Laird, "Meta-analysis in clinical trials," Controlled Clinical Trials, vol. 7, no. 3, pp. 177-188, 1986.

[25] J. P. Higgins, S. G. Thompson, J. J. Deeks, and D. G. Altman, "Measuring inconsistency in meta-analyses," BMJ, vol. 327, no. 7414, pp. 557-560, 2003.

[26] C. J. Eskey, P. M. Meyers, T. N. Nguyen et al., "Indications for the performance of intracranial endovascular neurointerventional procedures: a scientific statement from the American Heart Association," Circulation, vol. 137, no. 21, pp. e661e689, 2018.

[27] T. Wang, K. Yang, J. Luo et al., "Outcomes after stenting for symptomatic intracranial arterial stenosis: a systematic review and meta-analysis," Journal of Neurology, vol. 267, no. 3, pp. 581-590, 2020.

[28] C. P. Derdeyn, D. Fiorella, M. J. Lynn et al., "Mechanisms of stroke after intracranial angioplasty and stenting in the SAMMPRIS trial," Neurosurgery, vol. 72, no. 5, pp. 777-795, 2013.

[29] P. Gao, Z. Zhao, D. Wang et al., "China angioplasty and stenting for symptomatic intracranial severe stenosis (CASSISS): a new, prospective, multicenter, randomized controlled trial in China," Interventional Neuroradiology, vol. 21, no. 2, pp. 196-204, 2015.

[30] Y. Zhu, H. Zhang, Y. Zhang et al., "Endovascular metal devices for the treatment of cerebrovascular diseases," Advanced Materials, vol. 31, no. 8, article e1805452, 2019.

[31] M. J. Alexander, A. Zauner, J. C. Chaloupka et al., "WEAVE trial: final results in 152 on-label patients," Stroke, vol. 50, no. 4, pp. 889-894, 2019.

[32] M. J. Alexander, A. Zauner, R. Gupta et al., "The WOVEN trial: Wingspan one-year vascular events and neurologic outcomes," Journal of Neurointerventional Surgery, vol. 13, no. 4, pp. 307-310, 2021.

[33] D. Xu, C. Zhang, T. Wang et al., "Evaluation of enterprise stent-assisted coiling and telescoping stent technique as treatment of supraclinoid blister aneurysms of the internal carotid artery," World Neurosurgery, vol. 110, pp. e890-e896, 2018.

[34] S. Rohde, J. Seckinger, S. Hähnel, P. A. Ringleb, M. Bendszus, and M. Hartmann, "Stent design lowers angiographic but not clinical adverse events in stenting of symptomatic intracranial stenosis-results of a single center study with 100 consecutive patients," International Journal of Stroke, vol. 8, no. 2, pp. 87-94, 2013.

[35] T. Mori, M. Fukuoka, K. Kazita, and K. Mori, "Follow-up study after intracranial percutaneous transluminal cerebral balloon angioplasty," AJNR. American Journal of Neuroradiology, vol. 19, no. 8, pp. 1525-1533, 1998. 
[36] K. Kang, Y. Zhang, J. Shuai et al., "Balloon-mounted stenting for ICAS in a multicenter registry study in China: a comparison with the WEAVE/WOVEN trial," Journal of NeuroInterventional Surgery, 2020.

[37] S. Yaghi, P. Khatri, A. de Havenon et al., "Peri-procedural stroke or death in stenting of symptomatic severe intracranial stenosis," Journal of Neurointerventional Surgery, vol. 12, no. 4, pp. 374-379, 2020.

[38] K. Kang, F. Gao, D. Mo et al., "Outcome of endovascular recanalization for intracranial in-stent restenosis," Journal of Neurointerventional Surgery, vol. 12, no. 11, pp. 1094-1098, 2020.

[39] F. Siddiq, M. Z. Memon, G. Vazquez, A. Safdar, and A. I. Qureshi, "Comparison between primary angioplasty and stent placement for symptomatic intracranial atherosclerotic disease: meta-analysis of case series," Neurosurgery, vol. 65, no. 6, pp. 1024-1034, 2009.

[40] H. Balshem, M. Helfand, H. J. Schünemann et al., "GRADE guidelines: 3 . Rating the quality of evidence," Journal of Clinical Epidemiology, vol. 64, no. 4, pp. 401-406, 2011.

[41] G. H. Guyatt, A. D. Oxman, R. Kunz et al., "GRADE guidelines 6. Rating the quality of evidence-imprecision," Journal of Clinical Epidemiology, vol. 64, no. 12, pp. 1283-1293, 2011.

[42] M. H. Murad, B. A. Swiglo, A. N. Sidawy, E. Ascher, and V. M. Montori, "Methodology for clinical practice guidelines for the management of arteriovenous access," Journal of Vascular Surgery, vol. 48, no. 5, pp. S26-S30, 2008. 